\title{
Associação entre estado nutricional e níveis pressóricos de adolescentes
}

\author{
Association between nutritional status and blood pressure levels in adolescents \\ Asociación entre estado nutricional y niveles de presión de adolescentes
}

Recebido: 12/07/2021 | Revisado: 19/07/2021 | Aceito: 30/07/2021 | Publicado: 05/08/2021

\author{
Emanuelle de Sousa Ferreira \\ ORCID: https://orcid.org/0000-0002-9339-1279 \\ Universidade Federal do Piauí, Brasil \\ E-mail: emanuelleferreira55@gmail.com \\ Larissa Carvalho Ribeiro de Sá Lustosa \\ ORCID: https://orcid.org/0000-0003-2062-0648 \\ Universidade Federal do Piauí, Brasil \\ E-mail: larissacarvalho100@ hotmail.com \\ Layanne Cristina de Carvalho Lavôr \\ ORCID: https://orcid.org/0000-0003-3954-2870 \\ Universidade Federal do Piauí, Brasil \\ E-mail: layannecristina94@gmail.com \\ Larisse Monteles Nascimento \\ ORCID: https://orcid.org/0000-0001-7678-2107 \\ Universidade Federal do Piauí, Brasil \\ E-mail: monteles_larisse@ hotmail.com \\ Amanda Ferreira Mesquita de Oliveira \\ ORCID: https://orcid.org/0000-0003-1496-6722 \\ Universidade Federal do Piauí, Brasil \\ E-mail: amandamsqt@ hotmail.com \\ Karoline de Macêdo Gonçalves Frota \\ ORCID: https://orcid.org/0000-0002-9202-5672 \\ Universidade Federal do Piaú, Brasil \\ E-mail: karolfrota@ufpi.edu.br
}

\begin{abstract}
Resumo
Introdução: A hipertensão arterial sistêmica (HAS) tornou-se uma das mais preocupantes doenças crônicas não transmissíveis, consistindo num dos principais fatores associados ao desenvolvimento de doenças cardiovasculares e tem sido comum em adolescentes. Assim, sua deteç̧ão precoce pode evitar complicações futuras. Dados do Estudo de Riscos Cardiovasculares em Adolescentes (ERICA) indicaram que 24\% dos adolescentes brasileiros tinham pressão arterial (PA) elevada. A elevação de PA em crianças e adolescentes possui várias condições predisponentes (ambientais, socioeconômicas, sazonais e geográficas), sendo a obesidade uma das principais. Objetivo: Verificar a associação entre estado nutricional e presença de HAS em adolescentes das cidades de Teresina e Picos (Piauí, Brasil). Materiais e Métodos: Estudo transversal de base populacional com 353 adolescentes entre 10 a 19 anos. Consideraram-se dados demográficos, socioeconômicos, de etilismo, hábito de fumar, atividade física, pressóricos e antropométricos. Utilizaram-se os testes de Mann-Whitney e Qui-Quadrado para comparação de variáveis contínuas e categóricas, respectivamente. Empregou-se regressão de Poisson bruta e ajustada para verificar a associação entre estado nutricional e HAS. Resultados: Observou-se proporção de adolescentes com sobrepeso e obesidade de 17,9\% e 10,0\%, respectivamente, e prevalência de HAS de 7,9\%, sem diferenças entre os sexos. Constatou-se associação significativa, após ajustes, entre HAS com obesidade na amostra total e entre os sexos. Conclusão: Foi significativa a associação entre HAS e obesidade, demonstrando a importância da manutenção do estado nutricional adequado na adolescência.
\end{abstract}

Palavras-chave: Hipertensão arterial sistêmica; Sobrepeso; Obesidade; Adolescente.

\begin{abstract}
Introduction: Systemic arterial hypertension (SAH) has become one of the most worrying chronic non-communicable diseases, consisting of one of the main factors associated with the development of cardiovascular diseases and has been common in adolescents. Thus, its early detection can prevent future complications. Data from the Study of Cardiovascular Risks in Adolescents (ERICA) indicated that 24\% of Brazilian adolescents had high blood pressure (BP). The elevation of BP in children and adolescents has several predisposing conditions (environmental, socioeconomic, seasonal and geographic), obesity being one of the main ones. Objective: To verify the association between nutritional status and the presence of SAH in adolescents from the cities of Teresina and Picos (Piauí, Brazil). Materials and Methods: Cross-sectional population-based study with 353 adolescents aged 10 to 19 years. demographic, socioeconomic, alcohol consumption, smoking, physical activity, blood pressure and anthropometric
\end{abstract}


data. The Mann-Whitney and Chi-Square tests were used to compare continuous and categorical variables, respectively. Crude and adjusted Poisson regression was used to verify the association between nutritional status and SAH. Results: There was a proportion of overweight and obese adolescents of $17.9 \%$ and $10.0 \%$, respectively, and a prevalence of SAH of $7.9 \%$, with no differences between the sexes. There was a significant association, after adjustments, between SAH and obesity in the total sample and between sexes. Conclusion: The association between SAH and obesity was significant, demonstrating the importance of maintenance of adequate nutritional status in adolescence.

Keywords: Systemic arterial hypertension; Overweight; Obesity; Adolescent.

\section{Resumen}

Introducción: La hipertensión arterial sistémica (HSA) se ha convertido en una de las enfermedades crónicas no transmisibles más preocupantes, constituida por uno de los principales factores asociados al desarrollo de enfermedades cardiovasculares y ha sido común en adolescentes. Por tanto, su detección precoz puede prevenir futuras complicaciones. Los datos del Estudio de Riesgos Cardiovasculares en Adolescentes (ERICA) indicaron que el 24\% de los adolescentes brasileños tenían hipertensión arterial (PA). La elevación de la PA en niños y adolescentes tiene varias condiciones predisponentes (ambientales, socioeconómicas, estacionales y geográficas), siendo la obesidad una de las principales. Objetivo: Verificar la asociación entre el estado nutricional y la presencia de HSA en adolescentes de las ciudades de Teresina y Picos (Piauí, Brasil). Materiales y Métodos: Estudio poblacional transversal con 353 adolescentes de 10 a 19 años. Se consideraron datos demográficos, socioeconómicos, consumo de alcohol, tabaquismo, actividad física, presión arterial y antropométricos. Se utilizaron las pruebas de Mann-Whitney y Chi-Cuadrado para comparar variables continuas y categóricas, respectivamente. Se utilizó regresión de Poisson cruda y ajustada para verificar la asociación entre el estado nutricional y la HSA. Resultados: Hubo una proporción de adolescentes con sobrepeso y obesidad del 17,9\% y 10,0\%, respectivamente, y una prevalencia de HAS del 7,9\%, sin diferencias entre los sexos. Se encontró asociación significativa, después de ajustes, entre HAS y obesidad en la muestra total y entre sexos. Conclusión: La asociación entre HAS y obesidad fue significativa, demostrando la importancia de mantenimiento de un estado nutricional adecuado en la adolescencia.

Palabras clave: Hipertensión arterial sistémica; Exceso de peso; Obesidad; Adolescente.

\section{Introdução}

A Organização Mundial da Saúde (OMS) define a adolescência como um período entre dez e dezenove anos de idade. Este período é caracterizado mudanças no desenvolvimento fisiológico, psicossocial, emocional e comportamental dos indivíduos, abrindo margem para o estabelecimento de hábitos inadequados como o frequente consumo de alimentos ultraprocessados e baixa prática de atividade física (Bricarello et al., 2018; Das et al., 2017; Enes, Camargo \& Justino, 2019; Cureau et al., 2016). Esses e outros comportamentos de risco favorecem o desenvolvimento de fatores de risco cardiovascular e de doenças crônicas não transmissíveis (DCNT’s), como a obesidade (Ferreira et al., 2017; Bricarello et al., 2018).

Entre essas DCNT's, a hipertensão arterial sistêmica (HAS) tornou-se uma das mais preocupantes, consistindo num dos principais fatores associados ao desenvolvimento de doenças cardiovasculares e, devido aos fatores supracitados, tem sido comum em adolescentes. Assim, sua detecção precoce pode evitar complicações futuras (Reuter et al., 2019; Jardim et al., 2020).

Os resultados do Estudo de Riscos Cardiovasculares em Adolescentes (ERICA), realizado em 2016, indicaram que $24 \%$ dos adolescentes brasileiros tinham pressão arterial (PA) elevada (Bloch et al., 2016). A elevação de PA em crianças e adolescentes possui várias condições predisponentes (ambientais, socioeconômicas, sazonais e geográficas), sendo a obesidade uma das principais (Nika et al., 2019). Existem diversos mecanismos pelos quais a obesidade pode levar ao aumento da pressão arterial, dentre estes, destaca-se a indução da resistência à insulina, que por sua vez induz o pâncreas a secretar insulina. A hiperinsulinemia resultante, leva à exaustão pancreática e deficiência de insulina, estimulando a reabsorção de sódio dos rins e aumentando a retenção de sódio, levando ao aumento do volume de plasma circulante e consequente aumento da pressão arterial (Tanaka, 2019).

Dados da Pesquisa Nacional de Saúde do Escolar-PENSE (IBGE, 2015) demonstraram prevalências importantes de excesso de peso e obesidade de $20,5 \%$ e 6,4\%, respectivamente, em adolescentes do Nordeste brasileiro. Ademais, dados do ERICA (2016) demonstraram prevalência de HAS de 10,0\% e de 6,0\% de obesidade em adolescentes de Teresina (PI). 
Com isso, é fundamental elucidar a relação entre HAS e estado nutricional na adolescência de municípios do semiárido brasileiro, haja visto que nesses locais a prevalência de HAS em adolescentes tem sido preocupante. Assim, objetivou-se verificar a associação entre estado nutricional e presença de HAS em adolescentes de Teresina e Picos (PI).

\section{Metodologia}

\section{Amostra e tipo de estudo}

Estudo transversal de base populacional envolvendo adolescentes com idade entre 10 a 19 anos, realizado por meio de visitas domiciliares em Teresina e Picos. É parte da pesquisa "Inquérito de Saúde de Base Populacional nos municípios de Teresina e Picos (PI)", que foi conduzida no período de Setembro de 2018 a Março de 2020. De acordo com o último censo do Instituto Brasileiro de Geografia e Estatística (IBGE), Teresina possuía população de 814.230 pessoas e área territorial de 1.391,293 km² enquanto Picos possuía população de 73.414 pessoas e área territorial de 577,284km² (IBGE, 2011).

Para calcular o tamanho amostral, considerou-se a estratificação da população de Teresina e Picos, segundo a idade dos indivíduos, para ambos os sexos: crianças menores de 2 anos; de 3 a 4 anos; de 5 a 9 anos; adolescentes de 10 a 14 anos; de 15 a 19 anos; adultos de 20 a 59 anos e idosos com mais de 60 anos.

Considerando o número de domicílios particulares dos municípios estudados (IBGE, 2013), foi calculado o número médio de indivíduos em cada grupo etário por domicílio em cada cidade. Assim, para garantir que, no mínimo, 30 indivíduos de cada grupo etário participassem da amostra, foi calculado o número de domicílios necessários para cada grupo etário. Assim, o maior tamanho de amostra em número de domicílios foi para o grupo etário de 3-4 anos do sexo feminino, tanto em Teresina ( $\mathrm{n}=578$ domicílios), quanto em Picos ( $\mathrm{n}=620$ domić́lios). A partir disso, foi obtido o número esperado de indivíduos para cada grupo etário e sexo, correspondendo a 46 e 44 indivíduos de 0 a 2 anos, 31 e 30 indivíduos de 3 a 4 anos, 80 e 77 indivíduos de 5 a 9 anos, 92 e 90 indivíduos de 10 a 14 anos, 93 e 99 indivíduos de 15 a 19 anos, 567 e 684 indivíduos de 20 a 59 anos e 72 e 106 indivíduos com idade maior ou igual a 60 anos dos sexos masculino e feminino, respectivamente, na cidade de Teresina-PI. Em Picos-PI, o número esperado de indivíduos foi de 43 e 46 indivíduos de 0 a 2 anos, 31 e 30 indivíduos de 3 a 4 anos, 82 e 81 indivíduos de 5 a 9 anos, 95 e 92 indivíduos de 10 a 14 anos, 98 e 103 indivíduos de 15 a 19 anos, 571 e 664 de 20 a 59 anos e 83 e 115 indivíduos com idade maior ou igual a 60 anos dos sexos masculino e feminino, respectivamente

Considerando o número de indivíduos para cada faixa etária, foram realizados estudos por simulação do comportamento do Intervalo de confiança de 95\% (IC95\%) e do coeficiente de variação do erro-padrão da proporção - CV(p) para estimativas da proporção (p) variando de $10 \%$ a $70 \%$, segundo grupos etários, sexo e respectivos tamanhos amostrais. O número de indivíduos foi considerado satisfatório, pois permitiu estimativas da proporção com $\mathrm{CV}$ (p) ao redor de $20 \%$.

Considerando que no levantamento de dados poderiam ocorrer perdas, o tamanho da amostra final foi ajustado utilizando $\mathrm{n}=\mathrm{n} 0 / 0,80$, sendo $\mathrm{n} 0=$ tamanho da amostra inicial em número de domicílios e $\mathrm{n}=$ tamanho da amostra final em número de domicílios, admitindo-se uma taxa de resposta de 80\%, resultando em aproximadamente 750 domicílios em Teresina e 780 domicílios em Picos.

Ao final obteve-se amostra de 2.011 indivíduos de 10 a 92 anos de idade. Dentre esses havia 382 adolescentes nos dois municípios, dos quais 227 residiam em Teresina e 155 em Picos. Devido à ausência de dados de alguns participantes, a amostra final para este estudo foi de 353 adolescentes (Figura 1). 
Figura 1. Fluxograma de seleção da amostra. Teresina/Picos, 2019.

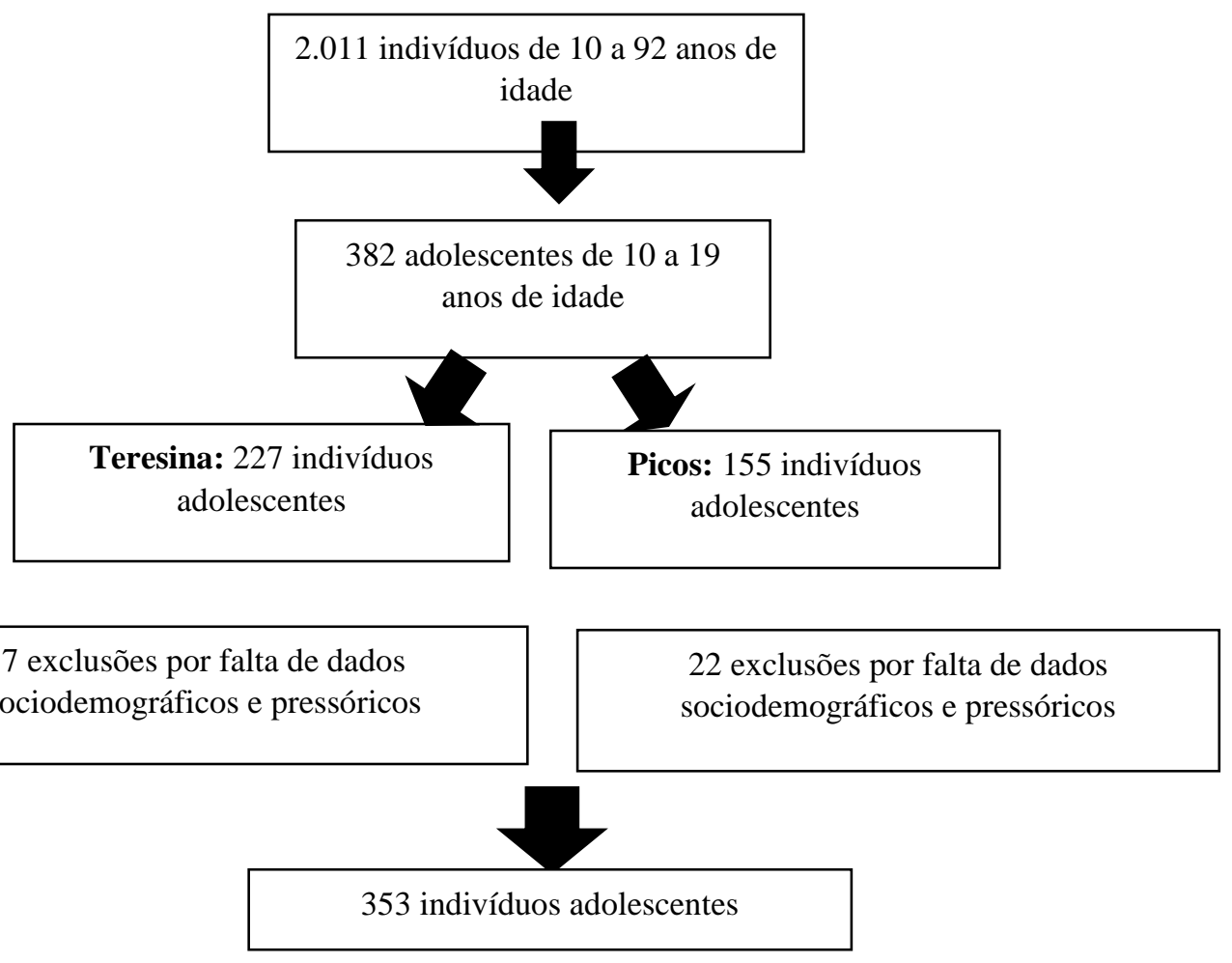

Fonte: Autores.

O plano de amostragem do estudo foi realizado por meio de processo de amostragem por conglomerados, em dois estágios: setores censitários e domicílios, com base nos dados do censo do IBGE para o ano de 2010.

O setor censitário é a menor unidade geográfica disponível para a qual existem dados dos residentes com características socioeconômicas semelhantes e é composto por aproximadamente 300 famílias (aproximadamente 1.000 habitantes).

Na primeira etapa, para melhorar a eficiência da amostragem, os setores censitários de cada cidade foram, quando necessário, divididos ou agrupados de tal forma que o coeficiente de variação para as suas dimensões não excedesse $10 \%$. Dessa forma, as Unidades Primárias de Amostragem (UPA’s) geradas poderiam ser constituídas por um único setor censitário, uma fração de um setor censitário, ou um agrupamento de setores censitários.

As UPA's foram, então, ordenadas de acordo com o seu código, de forma que todas as áreas da zona urbana dos municípios incluídos na pesquisa estivessem representadas na amostra. Assim, uma amostra sistemática foi tomada a partir desta lista ordenada das UPA's de cada cidade, com probabilidade proporcional ao tamanho.

A segunda etapa envolveu a amostragem sistemática de domicílios dentro de cada UPA selecionada na primeira etapa. Todos os moradores nos domicílios selecionados foram incluídos na pesquisa. A fração global de amostragem usada neste estudo foi: $f=\frac{a M_{i}}{\Sigma} M_{i} x \frac{b}{M_{i}}$ onde: $\mathrm{f}=$ fração global de amostragem; a=número total de UPA's a serem selecionadas no primeiro estágio; Mi=número de domicílios na UPA i; b=número de domicílios a serem sorteados em cada UPA selecionada. Assim, $f=\frac{n}{N}$ onde: $n=t a m a n h o$ da amostra em número de domicílios; $\mathrm{N}=$ tamanho da população em número de domicílios.

Desejando-se facilitar a estimação dos parâmetros de interesse, definiu-se que seriam sistematicamente selecionadas 30 UPA's em Teresina e 26 UPA's em Picos. Sendo assim, o número de domicílios a serem sorteados no segundo estágio de 
amostragem em cada UPA foi calculado como: $f=\frac{a M_{i}}{\sum M_{i}} x \frac{b}{M_{i}}=\frac{n}{N}$, o que resultou em 25 domicílios por UPA em Teresina e 26 domicílios por UPA em Picos.

Para variáveis que apresentavam valores faltantes optou-se por realizar a imputação pelo método Predictive Mean Matching, sendo que, o percentual de dados imputados não excedeu 20\%. Não houve ponderação dos dados, tendo em vista a probabilidade de seleção da amostra ter sido equiprobabilística. Todas as análises foram realizadas no módulo survey do Stata, considerando a amostragem complexa a fim de que os resultados obtidos fossem representativos da população total dos municípios pesquisados.

\section{Delineamento da pesquisa}

Os domicílios selecionados para a pesquisa foram visitados por equipe multiprofissional. A Figura 2 ilustra a ordem dos eventos para a realização da pesquisa.

Figura 2. Fluxograma de execução do estudo. Teresina/Picos, 2019.

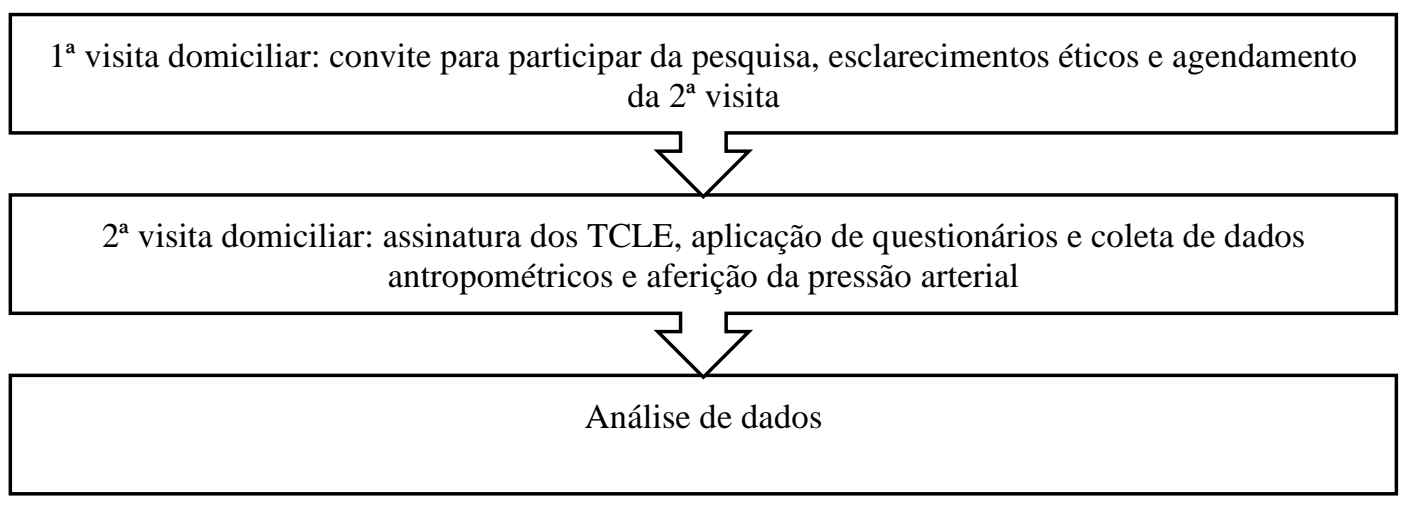

Fonte: Autores.

Para o presente estudo foram considerados dados demográficos (idade, sexo e cor da pele autorreferida), socioeconômicos (escolaridade e renda familiar), consumo de álcool, hábito de fumar, nível de atividade física, dados pressóricos e antropométricos.

A pesquisa foi aprovada pelo Comitê de Ética em Pesquisa da Universidade Federal do Piauí sob parecer de $n^{\circ}$ 2.522.426. Os adolescentes menores de 18 anos que concordaram em participar do estudo assinaram o Termo de Assentimento Livre e Esclarecido (TALE) e os pais ou responsáveis legais autorizaram a participação dos adolescentes na pesquisa mediante assinatura do Termo de Consentimento Livre e Esclarecido (TCLE). Adolescentes com idade $\geq 18$ anos assinaram o TCLE ao invés do TALE, sem a necessidade da autorização dos pais ou responsáveis legais, uma vez que já possuíam maioridade civil.

\section{Critérios de Inclusão e Exclusão}

Incluiu-se neste estudo adolescentes de ambos os sexos, com idade entre 10 e 19 anos, residentes em área urbana e em domicílios particulares permanentes no período de outubro de 2018 até dezembro de 2019 em Teresina e Picos. Foram considerados inelegíveis os residentes em domicílios coletivos, os que tinham deficiências ou incapacidades que dificultassem a aplicação dos questionários e/ou a avaliação antropométrica, além daqueles residentes em domicílios que permaneceram fechados após três visitas em dias alternados pela equipe de pesquisadores. 


\section{Procedimentos}

Os dados foram obtidos mediante aplicação de questionários estruturados, adaptados de estudos brasileiros como "ISA 2008: Inquéritos de Saúde na cidade de São Paulo" (Fisberg \& Marchioni, 2012), bem como daqueles utilizados na Pesquisa Nacional de Saúde realizada pelo IBGE (IBGE, 2013). Os questionários foram aplicados por meio do aplicativo Epicollect 5® (Imperial College London), utilizando-se dispositivos móveis (https://five.epicollect.net/project/isad).

Aferiu-se o peso utilizando balança eletrônica digital portátil (SECA ${ }^{\circledR}$, modelo 803, Hamburg, Germany) com capacidade de $150 \mathrm{~kg}$. A estatura foi medida com estadiômetro, desenvolvido pela equipe, desenvolvido segundo especificações do Laboratório de Avaliação do Estado Nutricional de Populações da Faculdade de Saúde Pública da Universidade de São Paulo (FSP/USP). Nas aferições os adolescentes estavam descalços, com os pés unidos, em posição ereta, olhando para o horizonte e vestindo roupas leves (Brasil, 2011). As medidas foram realizadas em duplicata e o valor médio utilizado para a análise.

Em seguida, o índice de massa corporal (IMC) foi calculado por meio da razão entre massa corporal em quilogramas e estatura em metros elevada ao quadrado $\left(\mathrm{kg} / \mathrm{m}^{2}\right)$. Para classificação do estado nutricional adotou-se as curvas propostas pela World Health Organization (WHO) em 2007 e adotados pelo Ministério da Saúde (Brasil, 2011), utilizando como índice o IMC-para-idade, segundo sexo. Assim, os pontos de corte adotados foram: escore- $Z<-2$ (magreza); escore-Z $\geq-2$ e $\leq 1$ (eutrofia); escore-Z $>1$ e $\leq 2$ (sobrepeso); escore- $Z>2$ (obesidade).

Dados sobre atividade física foram coletados por meio da versão em português do International Physical Activity Questionnaire (IPAQ) longo, o qual contém perguntas sobre duração, frequência e intensidade de atividades físicas ocupacionais, de lazer, domésticas e de transporte. Todos os domínios de prática de atividade física foram considerados para a obtenção do tempo total de atividade física leve a moderada e de prática de atividade física vigorosa por semana.

Os participantes foram classificados como "ativos" ou "inativos" considerando-se as recomendações para prática de atividade física da Organização Mundial da Saúde (OMS, 2010) que preconiza como adequada a prática de pelo menos 150 minutos de atividade física leve ou moderada e/ou 75 minutos de atividade física vigorosa por semana para indivíduos com idade igual ou acima de 18 anos. Para adolescentes de 10 a 17 anos, a recomendação considerada foi de 60 minutos diários ou 300 minutos de atividade física semanal, obtida pelo somatório do tempo semanal de todas as atividades realizadas na última semana.

A medida da PA foi realizada conforme a $7^{\mathrm{a}}$ Diretriz Brasileira de Hipertensão Arterial Sistêmica (Malachias et al., 2016), com auxílio de esfigmomanômetro aneróide (Durashock Welch Allyn-Tycos ${ }^{\circledR}$, NY, USA, Modelo DS-44) calibrado e manguito de tamanho apropriado à circunferência do braço do adolescente.

A aferição foi executada após descanso de 3 a 5 minutos, com os indivíduos sentados, relaxados, com o braço na altura do coração, apoiado e com a palma da mão voltada para cima. Foram realizadas duas medidas com intervalo de um minuto (Malachias et al., 2016).

Adolescentes menores de 18 anos de idade foram classificados como: normotensos, se pressão arterial sistólica (PAS) e pressão arterial diastólica (PAD) menores que valores do percentil (p) 90 para estatura, sexo e idade; pré-hipertensos, se PAS ou $\mathrm{PAD} \geq \mathrm{p} 90$ e $<$ p 95 ou com PA $\geq 120 / 80 \mathrm{mmHg}$ e p menor que 95; e, hipertensos, se PAS e/ou PAD correspondia ao p 95 ou superior. Indivíduos a partir de 18 anos de idade foram classificados em: normotensos, quando apresentavam PAS $\leq 120$ mmHg e/ou PAD $\leq 80$ mmHg; pré-hipertensos, quando exibiram PAS entre 121 e 139 mmHg e/ou PAD entre 81 e 89 mmHg; hipertensos, quando possuíam PAS e PAD $\geq 140$ e $90 \mathrm{mmHg}$, respectivamente (Malachias et al., 2016).

Foram coletados dados relativos ao sexo, idade, cor da pele, escolaridade e renda familiar. Quanto ao sexo, os participantes foram classificados em masculino e feminino. A idade foi expressa em anos e categorizada em 10-14 e 15-19 anos. A cor da pele foi categorizada em branca, preta, parda e outros (amarela e indígena). A escolaridade foi classificada em 
graus de instrução como não frequentou a escola, ensino fundamental, ensino médio e ensino superior. A renda familiar foi categorizada em $\leq 2$ salários mínimos e $>2$ salários mínimos, segundo valor vigente em 2019 de $\mathrm{R} \$ 954,00$..

As análises foram realizadas no programa Stata versão 13.0 (Stata Corporation, College Station, Estados Unidos, TX 13.0). As variáveis foram testadas quanto à distribuição e homocedasticidade pelos testes de Kolmogorov-Smirnov e de Levene. Variáveis contínuas assimétricas foram apresentadas como medianas e intervalos interquartis, utilizando-se o teste de MannWhitney para compará-las entre os sexos. Variáveis categóricas foram apresentadas em frequências absolutas e relativas, e comparadas pelo teste Qui-Quadrado de Pearson.

A associação entre estado nutricional e a presença de HAS foi analisada por meio do teste Qui-quadrado de Pearson e da regressão de Poisson, expressa em Razão de Prevalência, bruta e ajustada para idade, sexo, consumo de álcool, hábito de fumar e prática de atividade física. Adotaram-se intervalos de confiança de $95 \%$ e nível de significância de $5 \%$.

\section{Resultados}

Entre os 353 adolescentes estudados, 57,5\% pertenciam ao sexo feminino e possuíam mediana de idade de 14 anos, enquanto $42,5 \%$ eram do sexo masculino, com mediana de idade de 15 anos. A maioria se autodeclarou parda (58,1\%) e com renda familiar $\leq$ dois salários mínimos $(71,4 \%)$ (Tabela 1$)$. 
Tabela 1. Caracterização da amostra segundo variáveis sociodemográficas, econômicas, consumo de álcool, hábito de fumar, IMC, prática de atividade física e PA por sexo $(\mathrm{n}=353)$. Teresina/Picos, 2019.

\begin{tabular}{|c|c|c|c|c|}
\hline Variáveis & Total & Feminino & Masculino & $p$ \\
\hline \multirow[t]{2}{*}{ Idade (anos) } & $\begin{array}{l}\text { Mediana } \\
\text { (p25-p75) }\end{array}$ & $\begin{array}{l}\text { Mediana } \\
\text { (p25-p75) }\end{array}$ & $\begin{array}{l}\text { Mediana } \\
\text { (p25-p75) }\end{array}$ & \\
\hline & $15(12-17)$ & $14(12-17)$ & $15(12-17)$ & $0,87 * *$ \\
\hline Cor da pele & n $(\%)$ & n $(\%)$ & n $(\%)$ & \\
\hline Branca & $66(18,7)$ & $30(14,8)$ & $36(24,0)$ & \multirow{4}{*}{$0,17 * * *$} \\
\hline Negra & $54(15,3)$ & $33(16,2)$ & $21(14,0)$ & \\
\hline Parda & $205(58,1)$ & $124(61,1)$ & $81(54,0)$ & \\
\hline Outras & $28(7,9)$ & $16(7,9)$ & $12(8,0)$ & \\
\hline Renda familiar* & n $(\%)$ & n $(\%)$ & n $(\%)$ & \multirow{3}{*}{$0,62 * * *$} \\
\hline $\begin{array}{l}\leq 2 \text { salários } \\
\text { mínimos }\end{array}$ & $252(71,4)$ & $147(72,4)$ & $105(70,0)$ & \\
\hline > 2 salários mínimos & $101(28,6)$ & $56(27,6)$ & $45(30,0)$ & \\
\hline Escolaridade & n $(\%)$ & n $(\%)$ & n $(\%)$ & \\
\hline E. Fundamental & $205(58,1)$ & $122(60,1)$ & $83(55,3)$ & \\
\hline E. Médio & $126(36,7)$ & $70(34,5)$ & $56(37,3)$ & \\
\hline E. Superior & $22(6,2)$ & $11(5,4)$ & $11(7,4)$ & \\
\hline Consumo de álcool & n $(\%)$ & n $(\%)$ & n $(\%)$ & \\
\hline Sim & $33(9,3)$ & $14(6,9)$ & $19(12,7)$ & \multirow{2}{*}{$0,06 * * *$} \\
\hline Não & $320(90,7)$ & $189(93,1)$ & $131(87,3)$ & \\
\hline Hábito de fumar & n $(\%)$ & n $(\%)$ & n $(\%)$ & \\
\hline Sim & $7(2,0)$ & $3(1,5)$ & $4(2,7)$ & \multirow{2}{*}{$0,42 * * *$} \\
\hline Não & $346(98,0)$ & $200(98,5)$ & $146(97,3)$ & \\
\hline \multirow[t]{2}{*}{ IMC $\left(\mathbf{k g} / \mathrm{m}^{2}\right)$} & $\begin{array}{l}\text { Mediana } \\
\text { (p25-p75) }\end{array}$ & $\begin{array}{l}\text { Mediana } \\
(\text { p25-p75) }\end{array}$ & $\begin{array}{l}\text { Mediana } \\
\text { (p25-p75) }\end{array}$ & \\
\hline & $19,8(17,5-23,0)$ & $19,5(17,2-23,0)$ & $19,9(18,1-22,9)$ & $0,36^{* *}$ \\
\hline Atividade física & n $(\%)$ & n $(\%)$ & n $(\%)$ & \multirow{3}{*}{$0,004 * * *$} \\
\hline Inativos & $117(33,1)$ & $80(39,4)$ & $37(24,7)$ & \\
\hline Ativos & $236(66,9)$ & $123(60,6)$ & $113(75,3)$ & \\
\hline PA & $\begin{array}{l}\text { Mediana } \\
(\text { p25-p75) }\end{array}$ & $\begin{array}{l}\text { Mediana } \\
(\text { p25-p75) }\end{array}$ & $\begin{array}{l}\text { Mediana } \\
(\text { p25-p75) }\end{array}$ & \\
\hline PAS (mmHg) & $105,5(100-115)$ & $104(99-110)$ & $110(100-120)$ & $0,02 * *$ \\
\hline PAD (mmHg) & $70(61-75)$ & $70(60-75)$ & $70(61,5-76)$ & $0,75^{* *}$ \\
\hline
\end{tabular}

*Salário mínimo de referência: R \$998,00 (2019). ** Teste de Mann-Whitney. ***Teste de Qui-quadrado de Pearson. IMC: índice de massa corporal; PA: pressão arterial; PAS: pressão arterial sistólica; PAD: pressão arterial diastólica. Fonte: Autores. 
A maior parte dos pesquisados $(58,1 \%)$ relatou ter cursado/estar cursando ensino fundamental. Quanto ao uso de álcool e hábito de fumar, 90,7\% dos adolescentes referiram não consumir bebida alcóolica e $98 \%$ afirmaram não serem fumantes.

No tocante ao IMC, a mediana total e o intervalo interquartil do grupo estudado correspondeu à 19,8 kg/m² $(17,5$ 23,0), sem diferença significativa entre os sexos. Foram evidenciados resultados significativos relacionados à prática de atividade física, com maior prevalência de indivíduos ativos entre os meninos $(75,3 \%)$.

Quanto à PAS, verificou-se que meninos apresentaram significativamente maior mediana quando comparados às meninas, não havendo diferença entre os sexos em relação à PAD $(p>0,05)$.

Na Tabela 2 mostrou-se que a maioria dos adolescentes tinha estado nutricional adequado. Quanto ao excesso de peso, houve maior prevalência de sobrepeso (17,9\%) seguida da obesidade (10,0\%), nos dois sexos. A prevalência de HAS para a amostra total foi $7,9 \%$ e de pré-hipertensão foi $12,5 \%$. Não houve diferenças estatisticamente significativas entre os sexos em relação ao estado nutricional e prevalência de HAS.

Tabela 2. Prevalência dos adolescentes segundo estado nutricional e PA por sexo (n=353). Teresina/Picos, 2019.

\begin{tabular}{|c|c|c|c|c|}
\hline & Total & Meninas & Meninos & $p^{*}$ \\
\hline Estado Nutricional & n (\%) & n (\%) & n (\%) & \\
\hline Magreza & $13(3,7)$ & $7(3,5)$ & $6(4,0)$ & \\
\hline Eutrofia & $240(68,4)$ & $143(71,1)$ & $97(64,7)$ & \\
\hline Sobrepeso & $63(17,9)$ & $36(17,9)$ & $27(18,0)$ & 0,31 \\
\hline Obesidade & $35(10,0)$ & $15(7,5)$ & $20(13,3)$ & \\
\hline PA & n (\%) & n (\%) & n (\%) & \\
\hline Normal & $281(79,6)$ & $167(82,3)$ & $114(76,0)$ & \\
\hline Pré-hipertensão & $44(12,5)$ & $21(10,3)$ & $23(15,3)$ & 0,31 \\
\hline Hipertensão & $28(7,9)$ & $15(7,4)$ & $13(8,7)$ & \\
\hline
\end{tabular}

*Teste de Qui-quadrado de Pearson. $p<0,05$. PA: pressão arterial.

Fonte: Autores.

A Tabela 3 apresenta os resultados referentes à associação entre a presença de HAS e estado nutricional dos adolescentes total e por sexo. Verificou-se associação positiva e significativa entre o desfecho e a obesidade na população total e em ambos os sexos, de modo que adolescentes obesos apresentaram 3,25 vezes (IC95\%: 1,7-6,0) maior prevalência de HAS quando comparados aos eutróficos, após ajustes. Ademais, as meninas obesas apresentaram 5,1 (IC95\%: 2,7-9,9) maior prevalência de HAS, enquanto que os meninos obesos apresentaram 2,24 (IC95\%: 1,1-4,5) vezes maior prevalência de HAS em relação aos eutróficos, após ajustes. 
Tabela 3. Associação entre HAS e estado nutricional dos adolescentes segundo sexo. Teresina/Picos, 2019.

\begin{tabular}{|c|c|c|c|c|c|c|}
\hline \multirow{2}{*}{$\begin{array}{c}\text { Estado } \\
\text { Nutricional }\end{array}$} & \multicolumn{2}{|c|}{ Total } & \multicolumn{2}{|c|}{ Meninas } & \multicolumn{2}{|c|}{ Meninos } \\
\hline & RP (IC) p & $\mathbf{R P}(\mathbf{I C}) \mathbf{p}^{* *}$ & RP (IC) p & $\mathbf{R P}(\mathbf{I C}) \mathbf{p}^{* *}$ & RP (IC) p & $\mathbf{R P}(\mathbf{I C}) \mathbf{p}^{* *}$ \\
\hline & \multicolumn{6}{|c|}{ Hipertensão Arterial Sistêmica } \\
\hline Eutrofia & Ref. & Ref. & Ref. & Ref. & Ref. & Ref. \\
\hline \multirow{3}{*}{ Sobrepeso } & 1,66 & 1,58 & 2,00 & 2,26 & 1,36 & 1,35 \\
\hline & $(0,9-3,0)$ & $(0,8-2,9)$ & $(0,9-4,3)$ & $(0,9-5,2)$ & $(0,5-3,2)$ & $(0,5-3,4)$ \\
\hline & 0,08 & 0,13 & 0,07 & 0,05 & 0,46 & 0,52 \\
\hline \multirow{3}{*}{ Obesidade } & 3,40 & 3,25 & 5,6 & 5,1 & 2,02 & 2,24 \\
\hline & $(1,9-6,1)$ & $(1,7-6,0)$ & $(2,8-11,1)$ & $(2,7-9,9)$ & $(0,9-4,2)$ & $(1,1-4,5)$ \\
\hline & 0,001 & 0,001 & 0,001 & 0,001 & 0,05 & 0,02 \\
\hline
\end{tabular}

HAS: hipertensão arterial sistêmica; RP: Razão de Prevalência; IC: Intervalo de Confiança de 95\%. ** Ajustado para idade, consumo de álcool, hábito de fumar e prática de atividade física.

Fonte: Autores.

\section{Discussão}

Os resultados evidenciaram que a maioria dos adolescentes apresentou estado nutricional adequado, sendo relevante a prevalência de sobrepeso nos dois sexos. A prevalência de HAS para a amostra total foi 7,9\% e a de pré-hipertensão foi 12,5\%, não havendo diferença entre os sexos. Além disso, demonstrou-se associação estatisticamente significativa entre HAS e obesidade na amostra total e por sexo.

Em pesquisa com adolescentes de Teresina também houve maior prevalência de eutrofia, seguida do sobrepeso e da obesidade (Lustosa et al., 2019a). Quanto ao excesso de peso, o ERICA apresentou que o sobrepeso foi o mais relevante em termos de prevalência $(17,1 \%)$, assim como no presente estudo. No ERICA a prevalência de HAS foi superior à verificada nesse estudo $(9,6 \%)$ e os autores apontam prevalências mais baixas no Norte $(8,4 \%)$ e no Nordeste $(8,4 \%)$. Ressalta-se que a aferição da PA no ERICA foi realizada com monitor oscilométrico, diferente do presente estudo, podendo ser um dos fatores a justificar a diferença entre as prevalências (Bloch et al., 2016). Em estudo com adolescentes piauienses de escolas particulares de Picos, a prevalência de hipertensão foi 17,1\% para meninos e 17,6\% para meninas, superiores aos valores desta pesquisa (Lustosa et al., 2019b). Por outro lado, Negrão et al. (2018) constatou prevalência de hipertensão em adolescentes de Teresina inferior à do presente estudo (3,2\%). Variações nas prevalências de HAS nos estudos podem ter ocorrido devido a abrangência dos estudos (local ou regional) e diferenças metodológicas, ressaltando-se que o presente estudo abrangeu dois municípios piauienses.

Nascimento-Ferreira et al. (2017), demonstraram associação entre HAS e obesidade, com a obesidade aumentando o risco de HAS em adolescentes. Outros estudos também mostraram que crianças e adolescentes com IMC elevado (sobrepeso e obesidade) tinham níveis pressóricos mais altos que indivíduos com IMC normal e baixo (Wang et al., 2018; Nika et al., 2019). Betz et al. (2018) verificaram em indivíduos de 8-18 anos de idade relação positiva entre IMC e PA. Ademais, estudo realizado na cidade de Goiânia (GO) demonstrou que a obesidade esteve independentemente associada à HAS em adolescentes, em ambos os sexos (De Almeida et al., 2017). De modo similar, na presente pesquisa houve associação entre HAS e obesidade. Assim, os achados desta investigação fortalecem que as intervenções para reduzir a PA devem ser centradas na redução de gordura corporal e normalização do IMC. 
O aumento de IMC prediz aumento de gordura abdominal e de PA, que quando elevada durante a infância e adolescência é associada ao desenvolvimento precoce de aterosclerose e incremento do risco cardiovascular em adultos (Masocha, Monyeki \& Czuz, 2020). Portanto, em conjunto com os resultados desta pesquisa, reforça-se a importância do controle do peso corporal na adolescência a fim de evitar complicações cardiovasculares futuras.

$\mathrm{O}$ excesso de gordura corporal, especialmente a intra-abdominal, pode ocasionar aumento da atividade do sistema nervoso simpático (SNS), o qual promove maior reabsorção de sódio, resistência vascular periférica e assim elevação da PA (Bloch et al., 2016). A obesidade também possui efeito na regulação da PA pois os adipócitos sintetizam e liberam vários hormônios, como adiponectina, leptina e angiotensina, que ativam o SNS, sistema renina-angiotensina-aldosterona, estresse oxidativo e inflamação sistêmica, ocasionando diminuição da síntese de óxido nítrico. Essa redução causa disfunção endotelial e hipertrofia vascular, que são fatores de risco para HAS. Além disso, foi verificado que a leptina no hipotálamo e o excesso de fatores inflamatórios, como TNF- $\alpha$, possuem relação com o desenvolvimento da HAS relacionada à obesidade. Pela fisiologia normal a via leptina-melanocortinérgica e a via $\operatorname{TNF} \alpha$-glutamatérgica são ativadas separadamente para regular a PA, enquanto que na obesidade seus efeitos são sinergizados, o que contribui significativamente para a hipertensão patológica (Yu \& Cai, 2017; Werneck et al., 2018).

Tem sido proposto que a maturidade sexual pode afetar a PA por meio da estatura e da gordura corporal. Adolescentes com maturidade sexual precoce apresentam maior incremento no IMC, além de maior medida da circunferência da cintura, especialmente no sexo feminino. Desse modo, esses fatores que podem estar relacionados à HAS na adolescência (Oliveira, Frutuoso \& Gambardilla, 2014; Jardim et al., 2020). No entanto, dados relativos à maturidade sexual não foram obtidos nesta pesquisa.

Limitações do estudo envolveram o fato do delineamento ser transversal e assim, impossibilitar estabelecer relação de causalidade entre estado nutricional e HAS. Ademais, devido há algumas dificuldades durante a coleta, alguns dados faltantes de pressão arterial precisaram ser imputados. Além disso, não foram contempladas outras variáveis de confundimento, como consumo alimentar, maturidade sexual, estágio puberal e uso de contraceptivos, relacionadas a alterações de PA, cuja não inserção no estudo pode subestimar ou superestimar a associação do estado nutricional com o desfecho, por serem importantes variáveis de ajuste. Ainda assim, a fortaleza do estudo é que o mesmo envolveu amostra representativa e probabilística de adolescentes de 10 a 19 anos de idade domiciliados em Teresina e Picos; além disso foi possível esclarecer mais sobre a relação entre HAS e estado nutricional na adolescência, mostrando a necessidade de intervenções em saúde precoces para adequar o estado nutricional na adolescência a fim de prevenir/tratar alterações nos níveis pressóricos.

\section{Conclusão}

Neste estudo, parcela importante dos adolescentes tinham sobrepeso e HAS. A obesidade associou-se positivamente e significativamente à presença de HAS nos adolescentes. Diante disso, demonstrou-se de suma importância a manutenção de estado nutricional adequado já na adolescência e os resultados podem subsidiar intervenções em saúde e estudos posteriores do assunto. Sugerem-se então pesquisas longitudinais envolvendo mais fatores que possam estar relacionados com a HAS na adolescência. Ressalta-se a importância da prevenção e do tratamento do sobrepeso e da obesidade em adolescentes nos municípios brasileiros por meio de ações executadas por equipe multidisciplinar, contando com profissional de nutrição e de educação física, a fim de diminuir as chances de alterações pressóricas na adolescência e em fases futuras.

\section{Referências}

Betz, H. H., Eisenmann, J. C., Laurson, K. R., Dubose, K. D., Reeves, M. J., Carlson, J. J. \& Pfeiffer, K. A. (2018). Physical activity, BMI, and blood pressure in US youth: NHANES 2003-2006. Pediatric Exercise Science, 30(3), 418-425. 
Bricarello, P.L., Poltronieri, F., Fernandes, R., Retondario, A., de Moraes Trindade, E.B.S., de Vasconcelos, F de A.G. (2018). Effects of the Dietary Approach to Stop Hypertension (DASH) diet on blood pressure, overweight and obesity in adolescents: A systematic review. Clin Nutr ESPEN; 28:1-11.

Bloch, K. V., Klein, C. H., Szklo, M., Kuschnir, M. C. C., Abreu, G. A., Barufaldi, L. A., Veiga, G. V., Schaan, B. \& Silva, T. L. N. (2016). ERICA: prevalências de hipertensão e obesidade em adolescentes brasileiros. Revista de Saúde Pública, 50(supl 1), 1-13s.

Brasil. Ministério da Saúde. Secretaria de Atenção à Saúde. Departamento de Atenção Básica. Orientações para a coleta e análise de dados antropométricos em serviços de saúde: Norma Técnica do Sistema de Vigilância Alimentar e Nutricional - SISVAN / Ministério da Saúde, Secretaria de Atenção à Saúde, Departamento de Atenção Básica. Ministério da Saúde, 2011. 76 p: il. - (Série G. Estatística e Informação em Saúde).

Cureau, F. V., Silva, T. L. N., Bloch, K. V., Fujimori, E., Belfort, D. R., Carvalho, K. M. B., Leon, E. B., Vasconcellos, M. T. L., Ekelund, U. \& Schaan, B. D. (2016). ERICA: inatividade física no lazer em adolescentes brasileiros. Revista de Saúde Pública, 50 (supl 1), 1-11s..

Das, J. K., Salam, R. A., Thornburg, K. L., Prentice, A. M., Campisi, S., Lassi, Z. S., Koletzko, B., \& Bhutta, Z. A. (2017). Nutrition in adolescents: physiology, metabolism, and nutritional needs. Annals of the New York Academy of Sciences, 1393(1), 21-33.

De Almeida, M.M.S, Guimarães, R.A., Veiga Jardim, P.C.B, Sousa, A.L.L., De Souza, M. M. (2017) Association between arterial hypertension and nutritional status in adolescents from Goiânia, Goiás, Brazil. PLoS One, 12(12):1-8.

Enes, C. C., Camargo, C. M. \& Justino, M. I. C. (2019). Ultra-processed food consumption and obesity in adolescents. Revista de Nutrição, 32(1), e18170.

Ferreira, N. L., Claro, R. M., Mingoti, A. S. \& Lopes, A. C. S. (2017). Coexistence of risk behaviors for being overweight among Brazilian adolescents. Preventive Medicine, 100(1), 135-142.

Fisberg, R. M. \& Marchioni, D. M. L. (2012). Manual de Avaliação do Consumo Alimentar em estudos populacionais: a experiência do inquérito de saúde em São Paulo (ISA). São Paulo: Faculdade de Saúde Pública da Universidade de São Paulo.

IBGE. Instituto Brasileiro de Geografia e Estatística. Sinopse do Censo Demográfico 2010.

IBGE. Instituto Brasileiro de Geografia e Estatística. Pesquisa Nacional de Saúde 2013.

Jardim, T. V., Rosner, B., Bloch, K. V., Kuschinir, M. C. C., Szklo, M. \& Jardim P. C. V. (2020). Blood pressure reference values for Brazilian adolescents: data from the Study of Cardiovascular Risk in Adolescents (ERICA Study). Jornal de Pediatria, 96(2), 168-176.

IBGE. Instituto Brasileiro de Geografia e Estatística. Pesquisa nacional de saúde do escolar: 2015 / IBGE, Coordenação de População e Indicadores Sociais. IBGE, 2016. 132 p.

Lustosa, L. C. R. S., Nascimento, L. M., Lavôr, L. C. C., Gomes, K. R. O., Mascarenhas, M. D. M. \& Frota, K. M. G. (2019a). Metabolic syndrome in adolescents and its association with diet quality. Revista de Nutrição, 32(1), e190004.

Lustosa, L. C. R. S, Barros, K. R. B., Lima, L. H. O., Freire, J. A. P. \& Frota, K. M. G. Fatores de risco cardiovascular e presença de síndrome metabólica em adolescentes de escolas particulares (2019b). Adolescência \& Saúde, 16(4), 104-112.

Malachias, M. V. B., Souza, W. K. S. B., Plavnik, F. L., Rodrigues, C. I. S., Brandão, A. A., Neves, M. F. T., Bortolotto, L. A., Franco, R. J. S., Poli-deFigueiredo, C. E., Jardim, P. C. B. V., Amodeo, C., Barbosa, E. C. D., Koch, V., Gomes, M. A. M., Paula, R. B., Póvoa, R. M. S., Colombo, F. C., Ferreira Filho, S., Miranda, R.D., Machado, C. A., Nobre, F., Nogueira, A. R., Mion Júnior, D., Kaiser, S., Forjaz, C. L. M., Almeida, F. A., Martim, J. F. V., Sass, N., Drager, L. F., Muxfeldt, E., Bodanese, L. C., Feitosa, A. D., Malta, D., Fuchs, S., Magalhães, M. E., Oigman, W., Moreira Filho, O., Pierin, A. M. G. Feitosa, G. S., Bortolotto, M. R. F. L., Magalhães, L. B. N. C., Silva, A. C. S., Ribeiro, J. M., Borelli, F. A. O., Gus, M., Passarelli Júnior, O., Toledo, J. Y., Salles, G. F., Martins, L. C., Jardim, T. S. V., Guimarães, I. C. B., Antonello, I. C., Lima Júnior, E., Matsudo, V., Silva, G. V., Costa, L. S., Alessi, A., Scala, L. C. N., Coelho, E. B., Souza, D., Lopes, H. F., Gowdak, M. M. G., Cordeiro Júnior, A. C., Torloni, M. R., Klein, M. R. S. T, Nogueira, P. K., Lotaif, L. A. D., Rosito, G. B. A., Moreno Júnior, H. et al. (2016). $7^{\text {a }}$ Diretriz Brasileira de Hipertensão Arterial Sistêmica. Arquivos Brasileiros de Cardiologia, $107(3)$, 1-82.

Masocha, V., Monyeki, M. A. \& Czyz, S. (2020) Longitudinal relationships between changes in body composition and changes in selected metabolic risk factors (abdominal obesity and blood pressure) among South African adolescents. Peer Journal, 23(8), e9331.

Nascimento-Ferreira, M. V., Moraes, A. C. F., Rendo-Urtega, T., Forkert, E. C. O., Collese, T. S., Cucato, G. G., Reis, V. M. M., Torres-Leal, F. L., Moreno, L. A. \& Carvalho, H. B. (2017). Cross-sectional, school-based study of 14-19 year olds showed that raised blood pressure was associated with obesity and abdominal obesity. Acta Paediatrica, 106(3), 489-496.

Negrão, L. D., Aguiar, A. O., Sousa, C. R. N., Gomes, K. R. O. \& Moreira-Araújo, R. S. R. (2018). Pressão arterial e estado nutricional de adolescentes da rede pública e privada de Teresina-PI. Adolescência \& Saúde, 15(supl 1), 53-61.

Nika, T., Stabouli, S., Kollios, K., Papadopoulou-Legbelou, K., Printza, N., Antza, C., Papachristou, F. \& Kotsis, V. (2019). Obesity and season as determinants of high blood pressure in a school-based screening study. Journal of Human Hypertension, 33(4), $277-285$.

Oliveira, J. R., Frutuoso, M. F. P. \& Gambardella, A. M. D. (2014). Associação entre maturação sexual, excesso de peso e adiposidade central em crianças e adolescentes de duas escolas de São Paulo. Journal of Human Growth and Development, 24(2), 201-207.

Reuter, C. P., Rodrigues, S. T., Barbian, C. D., Silveira, J. F. C., Schneiders, L. B., Soares, S. S., Burgos, L. T. \& Burgos, M. S. (2019). High blood pressure in schoolchildren: associated sociodemographic and biochemical factors. Revista Portuguesa de Cardiologia, 38(3), $195-201$.

Wang, S., Zhu, Y., Cheng, Y. \& Zhang, Y. (2018). Profiles of blood pressure among children and adolescents with diferente body mass index categories in Shandong, China. Blood Pressure, 27(1), 56-61.

Werneck, A. O., Oyeyemi, A. L., Gerage, A. M., Cyrino, E. S., Szwarcwald, C. L., Sardinha, L. B., \& Silva, D. R. (2018). Does leisure-time physical activity 
Research, Society and Development, v. 10, n. 10, e57101018169, 2021

(CC BY 4.0) | ISSN 2525-3409 | DOI: http://dx.doi.org/10.33448/rsd-v10i10.18169

attenuate or eliminate the positive association between obesity and high blood pressure? Journal of Clinical Hypertension, 20(5), 959-966.

WHO. World Health Organization (2010). Global recommendations on physical activity for health. Genebra: WHO. 58p.

Yu, B. \& Cai, D. (2017). Neural Programmatic Role of Leptin, TNF $\alpha$, Melanocortin, and Glutamate in Blood Pressure Regulation vs Obesity-Related Hypertension in Male C57BL/6 Mice. Endocrinology, 158(6), 1766-1775. 